\title{
Digital Innovation: Telepsychiatry for Measuring Psychiatric Disorders
}

\author{
Naureen Akber Ali1 and Adeel Khoja ${ }^{2}$ \\ ${ }^{1}$ School of Nursing and Midwifery, The Aga Khan University, Karachi, Pakistan \\ 2Department of Medicine, The Aga Khan University, Karachi, Pakistan
}

Globally, individuals with mental disabilities are facing human rights emergencies due to violations of their basic needs in availing mental health services. Mental health disorders are the primary cause of disability around the world, accounting for a third of all years lived with disability and 8 million deaths annually. ${ }^{1}$ It is projected that in 2030, mental health disorders will become the second leading cause of disease burden globally; hence, the disability-adjusted life years (DALYs) linked to mental health disorders will most likely increase. ${ }^{2}$ Moreover, mood disorders, anxiety disorders (including particular phobias), and excessive use of alcohol are found to be the most prevalent disorders. ${ }^{3}$ A year-long prevalence of mental health disorder ranges between $9.6 \%$ to $27.8 \%$ in the general adult population. 3

According to World Health Organization (WHO), over $40 \%$ of the countries have no mental health policy and more than $30 \%$ have no mental health programme. Whereas, about $25 \%$ of the countries have no mental health regulations, ${ }^{4}$ and the magnitude of mental health burden is not equivalent to its demand. In more than $33 \%$ of the countries, less than $1 \%$ of the total health budget is assigned to mental health. In addition to this, there is just one psychiatrist available per 100,000 population in more than half the countries of the world; whereas, $40 \%$ of the countries have less than one hospital bed available per 10,000 population for mental disorders. 4 The WHO 2017 report on the burden of mental disorder specifies that worldwide $80 \%$ of the individuals suffering from mental disorders belong to low- and middle-income countries (LMIC) ${ }^{5}$

Low- and middle-income countries, particularly Pakistan, suffer from the burden of mental disorders, fundamentally because of fragmented healthcare systems, sociodemographic characteristics, poverty, lack of mental health professionals or limited resources, social rejection (stigma) and health-seeking behaviours of individuals. ${ }^{6}$ It is evident that mental disorders in Pakistan also extend to an appalling level in both the rural and urban populations with depression and anxiety disorders found

Correspondence to: Dr. Adeel Khoja, Department of Medicine, The Aga Khan University Hospital, Stadium Road, Karachi, Pakistan

E-mail: adeel.khoja@aku.edu

Received: December 18, 2019; Revised: January 21, 2020; Accepted: January 27, 2020 to be the highest, followed by bipolar, schizophrenia, psychosomatic disorders, obsessive compulsive disorder and post-traumatic stress disorder. 6 Besides this, mental diseases are also considered as dishonourable (social stigma) and are associated with supernatural/religious causes for which people opt for faith-healers. ${ }^{6}$ Thus, societal perception and lack of awareness regarding mental disorders further affect the community, and adds substantial disease burden.

Digital technology, like telepsychiatry, provides a feasible solution, as it offers the delivery of mental healthcare across distances via utilising communication technologies like telephones, videoconferencing, and electronic health records. Telepsychiatry offers a novel opportunity that improves patients' access to qualified mental healthcare professionals that saves their time and cost. ${ }^{7}$ In developed countries like US, the proportion of telepsychiatry services have increased considerably from $15.2 \%$ in 2010 to $29.2 \%$ in 2017.7 A systematic review also showed that telepsychiatry services enhance patient's access to care, sustain patient/family satisfaction, reduce service cost and is found to be feasible and accessible for the clinical team. 8 Another study identified that provision of telepsychiatry services resulted in change in the diagnostic therapy and treatment regimen for the patients. Moreover, after a telepsychiatry consultation, majority of the patients $(72.3 \%)$ showed significant clinical improvement. 9 Furthermore, telepsychiatry care has the potential to leverage limited mental health resources to target more patients, thus permitting mental health experts to have a greater impact at population level as compared to traditional method of care, i.e. face-to-face consultation. ${ }^{10}$ Hence, telepsychiatry mode is considered a valid and reliable tool for dealing with psychiatric patients living in far-flung areas. 7

Telepsychiatry has breached all the geospatial boundaries; but in developing nations such as Pakistan, telepsychiatry services are still in its infancy stage and need to expand particularly in the remote areas of Pakistan that are difficult to access. A telepsychiatry pilot project has been initiated in 2015 in Gilgit and Chitral for flood victims, where participants diagnosed with posttraumatic stress disorder (PTSD) and/or depression, underwent telepsychiatry-based sessions with a qualified psychiatrist at least once a week. ${ }^{11}$ Another project launched by the Aga Khan Development Network 
in Northern areas offers telepsychiatry services near to patient's home. This service reduces their cost in terms of travel expenses, time, and lodging which increases their satisfaction level. Besides, almost $99 \%$ of the participants shared that they will recommend these services to other psychiatric patients. ${ }^{12}$

Current situation predicts that in the near future, demand for mental healthcare services will remain high as compared to the supply of providers (demand and supply gap) which calls for an integration of innovative practices like introducing telepsychiatry services into the mental healthcare model. Though, there is a rising trend of using telepsychiatry innovations in low-income setting like Pakistan; but still, there is a need to expand this highly effective technology in remote areas having different socio-cultural barriers. Hence, telepsychiatry service improves patient's access to quality mental health services that include: permitting distant care delivery and access to mental health professionals along with capacity-building of junior local healthcare providers, enabling effective utilisation of cost and time.

At the policy level, telepsychiatry services should be promoted and introduced by the government in the healthcare system for effective handling of mental health cases that can minimise the treatment gap for vulnerable/isolated communities to seek mental health expert consultations. Additionally, receiving timely treatment and much needed attention via telepsychiatry can reduce the existing mental healthcare burden across hard-to-reach areas. Moreover, in future a hybrid model where a combination of both in-person (face-toface) and technology-based care should be integrated in the healthcare system for patients having mental illnesses.

Lastly, further research is needed to provide effective models by intervening telepsychiatry-based practice in primary care settings that ultimately targets larger remote communities.

\section{REFERENCES}

1. Walker ER, McGee RE, Druss BG. Mortality in mental disorders and global disease burden implications: A systematic review and meta-analysis. JAMA Psychiatry 2015; 72:334-41.

2. Veisani $Y$, Delpisheh A, Mohamadian F. Prevalence and gender differences in psychiatric disorders and DSM-IV mental disorders: a population-based study. Biomed Res Ther 2018; 5:1975-85.

3. Gustavson K, Knudsen AK, Nesvåg R, Knudsen GP, Vollset SE, Reichborn-Kjennerud T. Prevalence and stability of mental disorders among young adults: Findings from a longitudinal study. BMC Psychiatry 2018; 18:65.

4. World Health Organization (WHO, 2001). World health report. Mental disorders affect one in four people. Available online: https://www.who.int/whr/2001/media_centre/press_release/en/

5. Opio JN, Tufanaru C, Aromataris E. Prevalence of mental disorders in Uganda: A systematic review protocol. JBI Database System Rev Implement Rep 2018; 16:1613-20.

6. Khalily MT. Mental health problems in Pakistani society as a consequence of violence and trauma: A case for better integration of care. Int J Integr Care 2011; 11:e128.

7. Spivak S, Spivak A, Cullen B, Meuchel J, Johnston D, Chernow R, et al. Telepsychiatry use in US mental health facilities, 20102017. Psychiatr Serv 2019; 201900261.

8. O'Keefe M, White K, Jennings JAC. Asynchronous telepsychiatry: A systematic review. J Telemed Telecare 2019; $1357633 X 19867189$.

9. Marcin JP, Nesbitt TS, Cole SL, Knuttel RM, Hilty DM, Prescott PT, et al. Changes in diagnosis, treatment, and clinical improvement among patients receiving telemedicine consultations. Telemed J E-Health 2005; 11:36-43.

10. Fortney JC, Pyne JM, Turner EE, Farris KM, Normoyle TM, Avery MD, et al. Telepsychiatry integration of mental health services into rural primary care settings. Int Rev Psychiatry 2015; 27:525-39.

11. Qadir TF, Fatima H, Usmani MH, Hussain SA. Telepsychiatry in Pakistan after natural disasters. Lancet Psychiatry 2016; 3: 1016.

12. Impact evaluation: Assessing the effectiveness of telepsychiatry consultations in Pakistan. (2018) Available online: https:// www.akdn.org/what-we-do/health/ehealth-resources-centre/ effectiveness-telepsychiatry-consultations. 